\title{
THE SEMISIMPLICITY PROBLEM FOR $p$-ADIC GROUP ALGEBRAS
}

\author{
KATHRYN E. HARE AND MAZIAR SHIRVANI
}

(Communicated by Warren J. Wong)

\begin{abstract}
For a prime $p$ let $\Omega=\Omega_{p}$ denote the completion of the algebraic closure of the field of $p$-adic numbers with $p$-adic valuation $|\cdot|$. Given a group $G$ consider the ring of formal sums

$$
l_{1}(\Omega, G)=\left\{\sum_{x \in G} \alpha_{x} x: \alpha_{x} \in \Omega,\left|\alpha_{x}\right| \rightarrow 0\right\} .
$$

Motivated by the study of group rings and the complex Banach algebras $l_{1}(\mathbf{C}, G)$, we consider the problem of when this ring is semisimple (semiprimitive). Our main result is that for an Abelian group $G, l_{1}(\Omega, G)$ is semisimple if and only if $G$ does not contain a $C_{p} \infty$ subgroup. We also prove that $l_{1}(\Omega, G)$ is semisimple if $G$ is a nilpotent $p^{\prime}$-group, an ordered group, or a torsion-free solvable group. We use a mixture of algebraic and analytic methods.
\end{abstract}

\section{INTRODUCTION}

Throughout $p$ is a fixed prime, and all fields are contained in $\Omega=\Omega_{p}$, the completion of the algebraic closure of the field of $p$-adic numbers $\mathbf{Q}_{p}$, and contain $\mathbf{Q}_{p}$. If $k$ is such a complete field denote by $|\cdot|: k \rightarrow \mathbf{R}$ the non-archimedean extension of the $p$-adic valuation on $\mathbf{Q}_{p}$. Given a group $G$ consider the ring of formal sums

$$
l_{1}(k, G)=\left\{\sum_{x \in G} \alpha_{x} x: \alpha_{x} \in k,\left|\alpha_{x}\right| \rightarrow 0\right\}
$$

where $\left|\alpha_{x}\right| \rightarrow 0$ means that for every $\varepsilon>0$, only finitely many of the $\alpha_{x}$ satisfy $\left|\alpha_{x}\right| \geq \varepsilon$. We are interested in the problem of whether the ring, $l_{1}(k, G)$, is semisimple (sometimes called semiprimitive) or not. Our main results are that for Abelian groups $G, l_{1}(k, G)$ is semisimple if $G$ does not contain a $C_{p^{\infty}}$

Received by the editors April 8, 1989 and, in revised form, May 24, 1989.

1980 Mathematics Subject Classification (1985 Revision). Primary 16A20; Secondary 16A27, 26E30.

The first author's research was partially supported by NSERC of Canada and the University of Waterloo.

The second author's research was partially supported by NSERC of Canada.

(C) 1990 American Mathematical Society $0002-9939 / 90 \$ 1.00+\$ .25$ per page 
subgroup (§3), and that for a large class of solvable groups including all nilpotent $p^{\prime}$-groups, $l_{1}(\Omega, G)$ is again semisimple $(\S 4)$.

The algebra $l_{1}\left(\mathbf{Q}_{p}, G\right)$ was studied in [10]. They consider the question of when $l_{1}\left(\mathbf{Q}_{p}, G\right)$ is Noetherian, Artinian, or prime and ask when it is semiprime. Our work was also motivated by the well-known result that the Banach algebra over C

$$
l_{1}(G)=\left\{\sum_{x \in G} \alpha_{x} x: \alpha_{x} \in \mathbf{C}, \sum\left|\alpha_{x}\right|<\infty\right\}
$$

(here $|\cdot|$ denotes the usual absolute value on $\mathbf{C}$ ) is always semisimple (cf. [8] or [13]). In contrast it is known that $l_{1}\left(\Omega, C_{p^{\infty}}\right)$ is not semisimple [4].

We conclude the paper with a list of some open problems.

\section{General Results}

Define $\|\cdot\|: l_{1}(k, G) \rightarrow \mathbf{R}$ as follows: if $f=\sum \alpha_{x} x \in l_{1}(k, G)$ then $\|f\|=$ $\sup _{x \in G}\left|\alpha_{x}\right|$. Also, write $\operatorname{supp}(f)=\left\{x \in G: \alpha_{x} \neq 0\right\}$. In the following lemma we collect a number of (very easy to prove) properties of $l_{1}(k, G)$ :

2.1. Lemma. Let $k$ be a complete subfield of $\Omega$ containing $\mathbf{Q}_{p}$ and let $G$ be a group. Then:

(i) If $f \in l_{1}(k, G)$ then $\operatorname{supp}(f)$ is countable.

(ii) $\|f\|=\max _{x \in G}\left|\alpha_{x}\right|$ if $f=\sum \alpha_{x} x$, and we have:

$\|f\| \geq 0$ for all $f$, and $\|f\|=0$ if and only if $f=0$;

$\|f+g\| \leq \max \{\|f\|,\|g\|\}$ and $\|f+g\|=\|f\|$ if $\|g\|<\|f\|$;

$\|f g\| \leq\|f\|\|g\|$.

In particular the topology defined by $\|\cdot\|$ turns $l_{1}(k, G)$ into an ultrametric topological ring which is complete.

(iii) The maximal right (or left ideals) of $l_{1}(k, G)$ are closed.

(iv) Let $\varphi: l_{1}(k, G) \rightarrow \Omega$ be a k-algebra homomorphism. Then $\varphi$ is continuous if and only if $|\varphi(f)| \leq\|f\|$ for all $f \in l_{1}(k, G)$.

(v) Let $\varphi: G \rightarrow \Omega$ be a k-algebra homomorphism where $|\varphi(x)|=1$ for all $x \in G$. Then $\varphi$ has a unique continuous extension to $l_{1}(k, G)$.

Proof. (i) and (ii) are trivial. As for (iii), let $M$ be a maximal right ideal of $l_{1}(k, G)$. Then its closure $\bar{M}$ is also a right ideal. If $1 \in \bar{M}$ then we can find a sequence $\left\{f_{n}\right\}$ of elements of $M$ with $f_{n} \rightarrow 1$. But if $\left\|1-f_{n}\right\|<1$ then $f_{n}=1-\left(1-f_{n}\right)$ is a unit (with inverse $\sum_{i=0}^{\infty}\left(1-f_{n}\right)^{i} \in l_{1}(k, G)$ since $l_{1}(k, G)$ is complete). This is impossible, so $1 \notin \bar{M}$ and so $\bar{M}=M$.

(iv) Assume $\varphi$ is continuous and suppose there exists $x \in G$ with $|\varphi(x)| \neq$ 1. Choose $\alpha \in k$ with $0<|\alpha|<1$. Replacing $x$ by a suitable power we may assume that $|\varphi(x)|>\alpha^{-1}$. Consider the element $f=\sum_{i=0}^{\infty} \alpha^{i} x^{i} \in l_{1}(k, G)$. If $f_{n}=\sum_{i=0}^{n} \alpha^{i} x^{i}$ then clearly $\left\|f-f_{n}\right\| \rightarrow 0$, while $\left|\varphi\left(f_{n}\right)-\varphi\left(f_{n-1}\right)\right|=$ $\left|\alpha^{n} \varphi(x)^{n}\right| \rightarrow \infty$, so the sequence $\left\{\varphi\left(f_{n}\right)\right\}$ is not convergent in $\Omega$. This contra- 
dicts the continuity of $\varphi$. Thus $|\varphi(x)|=1$ for all $x \in G$. Let $f=\sum_{x \in G} a_{x} x \in$ $l_{1}(k, G)$. By continuity $\varphi(f)=\sum a_{x} \varphi(x)$, thus $|\varphi(f)| \leq\|f\|$. The other direction is trivial.

(v) It is easy to check that the unique continuous extension is given by $\varphi\left(\sum a_{x} x\right)=\sum a_{x} \varphi(x)$.

If $R$ is a ring we write $J(R)$ for the Jacobson radical of $R$. The following result enables us to concentrate on countable groups:

2.2. Lemma. If $k$ is a complete field and $G$ is a group, then

$$
J\left(l_{1}(k, G)\right) \subseteq \bigcup_{H} J\left(l_{1}(k, H)\right),
$$

where the union ranges over all countable subgroups $H$ of $G$.

Proof. Let $f \in J\left(l_{1}(k, G)\right)$ and let $H=\langle\operatorname{supp}(f)\rangle$. We claim that $f \in$ $J\left(l_{1}(k, H)\right)$. If $g \in l_{1}(k, H)$ then there exists $f^{\prime} \in l_{1}(k, G)$ with $f^{\prime}(1-g f)=$ 1. Let $T$ be a left transversal of $H$ to $G$ containing 1 , and write $f^{\prime}=\sum t f_{t}^{\prime}$, where $f_{t}^{\prime} \in l_{1}(k, H)$. It follows from $\sum_{t \in T} t f_{t}^{\prime}(1-g f)=1$ that $f_{1}^{\prime}(1-g f)=1$. This is for all $g \in l_{1}(k, H)$, so $f \in J\left(l_{1}(k, H)\right)$, as required.

If $k$ is a field write $X_{k}$ for the class of all groups $G$ for which $l_{1}(k, G)$ is semisimple. Not much is known about the class-theoretic properties of $X_{k}$. As an example we have:

2.3. Theorem. Let $k$ be a complete field, and let $\left\{N_{\lambda}: \lambda \in \Lambda\right\}$ be a directed system of normal subgroups of $G$ such that every $G / N_{\lambda} \in X_{k}$. Then $G \in X_{k}$.

Proof. Let $0 \neq f \in l_{1}(k, G)$, and write $f=\sum_{i=1}^{r} \alpha_{i} x_{i}+g$ where $\left|\alpha_{i}\right|=\|f\|$ for all $i=1, \ldots, r$ and $\|g\|<\|f\|$. By assumption there exists $\lambda \in \Lambda$ with $x_{i} x_{j}^{-i} \notin N_{\lambda}$ for $1 \leq i \neq j \leq r$. The mapping $\theta: l_{1}(k, G) \rightarrow l_{1}\left(k, G / N_{\lambda}\right)$ obtained by extending the natural homomorphism $G \rightarrow G / N_{\lambda}$ is easily seen to be a well-defined surjective continuous ring homomorphism. Now $\theta(f)=$ $\sum \alpha_{i} \theta\left(x_{i}\right)+\theta(g)$, the elements $\theta\left(x_{i}\right)$ are distinct, and $\|\theta(g)\| \leq\|g\|<\|f\|$. Thus $\theta(f) \neq 0$, and since $J\left(l_{1}\left(k, G / N_{\lambda}\right)\right)=\{0\}$ this implies that $f \notin$ $J\left(l_{1}(k, G)\right)$, as required.

2.4. Corollary. If $G$ is residually finite then $G \in X_{k}$ for all $k$.

Proof. If $N \triangleleft_{f} G$ then $l_{1}(k, G / N)$ is simply the group ring of $G / N$ over $k$, which is semisimple by Maschke's Theorem ([11], 2.4.2).

As another example we have

2.5. Theorem. Let $k$ be complete, with $\bar{k}$ the residue class field of $k$. If the group ring $\bar{k} G$ is semisimple and has no zero divisors then $G \in X_{k}$. 
Proof. Suppose $J=J\left(l_{1}(k, G)\right) \neq 0$, so it contains an element $g$ with $\|g\|=$ 1. Choose any $f \in l_{1}(k, G)$ with $\|f\|=1$. If $\|1-f g\|<1$ then $f g=$ $1-(1-f g)$ is invertible, which is impossible since $g \in J$. Let $u \in l_{1}(k, G)$ be such that $u(1-f g)=1$. We claim that $\|u\|=1$. First, note that

$$
1=\|u(1-f g)\| \leq\|u\|\|1-f g\|=\|u\| \text {. }
$$

Now set

$$
\begin{gathered}
R=\left\{y \in l_{1}(k, G):\|y\| \leq 1\right\}, \\
M=\left\{y \in l_{1}(k, G):\|y\|<1\right\} .
\end{gathered}
$$

Then $M$ is an ideal of $R$ and $R / M \cong \bar{k} G$ (for the coefficients of the elements of $R$ belong to the valuation ring of $k$, and the homomorphism onto $\bar{k}$ is easily seen to extend to one of $R$ onto $\bar{k} G$ with kernel $M)$. Let $\pi: R \rightarrow R / M$ denote the canonical map. If $\|u\|>1$ choose $\alpha \in k$ with $|\alpha|=\|u\|$. Then $\left(\alpha^{-1} u\right)(1-f g)=\alpha^{-1}$, and so $\left(\alpha^{-1} u\right) \pi(1-f g) \pi=\alpha^{-1} \pi=0$. But this is impossible since $\left\|\alpha^{-1} u\right\|=\|1-f g\|=1$ and $\bar{k} G$ has no zero divisors. Thus $\|u\|=1$. But then $(u \pi)(1-f \pi g \pi)=1$, and since this is true for all $f \in l_{1}(k, G)$ it follows that $g \pi \in J(\bar{k} G)=\{0\}$, contradicting $\|g\|=1$.

2.6. Corollary. Let $k$ be a complete field.

(i) If $G$ is a u.p. group (e.g. an ordered group) then $G \in X_{k}$.

(ii) If $\bar{k}$ is uncountable and $\bar{k} G$ has no zero divisors then $G \in X_{k}$.

Proof. These are consequences of 2.5 and well-known facts about the groupring $\bar{k} G$ (cf. [11], 13.1.2, 13.1.9, 7.1.6) in view of the fact that u.p. groups are t.u.p. groups [14].

2.7. Corollary. Let $k$ be complete with residue class field $\bar{k}$, and let $G$ and $H$ be nontrivial groups. If the group ring $\bar{k}(G \times H)$ has no zero divisors then $G \times H \in X_{k}$.

Proof. The assumptions imply that $\bar{k}(G \times H)$ is semisimple [9]. The result follows from 2.5 .

2.8. Corollary. Let $k$ be complete with residue class field $\bar{k}$. Suppose $G$ is a torsion-free solvable group. Then $G \in X_{k}$.

Proof. In [7] it is shown that for such groups $G, \bar{k}(G)$ has no zero divisors. It is well known that $\bar{k}(G)$ is semisimple (cf. [11] 7.4.6).

\section{ABELIAN GROUPS}

For $f \in l_{1}(k, G)$ let $v(f)=\lim _{n \rightarrow \infty}\left\|f^{n}\right\|^{1 / n}$ (it is easy to see that $v(f)$ is well defined, [12] 6.22). Say $f$ is topologically nilpotent if $v(f)=0$. Our aim is to show that for Abelian groups $G$, the Jacobson radical of $l_{1}(k, G)$ is precisely the set of topological nilpotents in $l_{1}(k, G)$, as is the case for the 
complex algebra $l_{1}(G)$ [8]. We will write $\Phi_{k}(G)$ for the set of all continuous $k$-algebra homomorphisms $\varphi: l_{1}(k, G) \rightarrow \Omega$ and write $\Phi_{k}^{\prime}(G)$ for the set of $\varphi \in \Phi_{k}(G)$ such that $\varphi\left(l_{1}(k, G)\right)$ is a field. We have:

3.1. Lemma. Let $G$ be an Abelian group, with $H$ a subgroup of $G$. Then any element of $\Phi_{k}(H)$ (respectively, $\Phi_{k}^{\prime}(H)$ ) can be extended to an element of $\Phi_{k}(G)$ (respectively, $\Phi_{k}^{\prime}(G)$ ).

Proof. Apply Zorn's Lemma to the set of all triplets $\left(G_{1}, \varphi, E\right)$ where $G_{1} \supseteq H$ is a subgroup of $G, \varphi \in \Phi_{k}\left(G_{1}\right)$, (respectively, $\Phi_{k}^{\prime}\left(G_{1}\right)$ ), and $E=$ $\varphi\left(l_{1}\left(k, G_{1}\right)\right)$. It is routine to show that $G$ belongs to a maximal element (cf. [11] 1.2.7).

We need to introduce an auxiliary seminorm on $l_{1}(k, G)$ : if $f \in l_{1}(k, G)$ let

$$
\|f\|_{s p}=\sup \left\{|\varphi(f)|: \varphi \in \Phi_{k}^{\prime}(G)\right\} .
$$

We have the following result:

3.2. Lemma. Let $k$ be a complete field, and let $G$ be an Abelian group.

(a) $v(f+g) \leq \max \{v(f), v(g)\}$ for all $f, g \in l_{1}(k, G)$.

(b) $v(f) \leq\|f\|$ for all $f \in l_{1}(k, G)$.

(c) $v$ is continuous.

(d) $\|f\|_{s p} \leq v(f) \leq p\|f\|_{s p}$ for all $f \in l_{1}(k, G)$.

Proof. For (a) see [12], 6.22. Part (b) is obvious. Part (c) follows easily from (a) and (b). Consider (d). The inequality $\|f\|_{s p} \leq v(f)$ follows from $\left\|f^{n}\right\|_{s p} \leq$ $\left\|f^{n}\right\|$. To prove the second inequality, first consider the case when $G$ is finitely generated, and let $\lambda \in k$ satisfy $\|f\|_{s p}<|\lambda|$. Then $\left|\varphi\left(\lambda^{-1} f\right)\right|=\left|\lambda^{-1}\right||\varphi(f)| \leq$ $|\lambda|^{-1}\|f\|_{s p}<1$ for all $\varphi \in \Phi_{k}^{\prime}$, and so $\left(\lambda^{-1} f\right)^{n} \rightarrow 0$ as $n \rightarrow \infty$ [15]. Thus for all sufficiently large $n$ we have $\left\|\left(\lambda^{-1} f\right)^{n}\right\|<1$, whence $\left\|f^{n}\right\|^{1 / n}<|\lambda|$. Thus $v(f) \leq|\lambda|$ whenever $\lambda \in k$ satisfies $\|f\|_{s p}<|\lambda|$. Since $\left|k^{*}\right| \supseteq\left\{p^{n}: n \in \mathbf{Z}\right\}$, and in particular has 0 as an accumulation point, it follows that $v(f)=0$ if $\|f\|_{s p}=0$. It also follows that if $\|f\|_{s p} \neq 0$ then there exists $\lambda \in k$, with $\|f\|_{s p}<|\lambda| \leq p\|f\|_{s p}$, and hence $v(f) \leq p\|f\|_{s p}$.

In the general case write $f=\lim _{n \rightarrow \infty} f_{n}$ where each $f_{n} \in l_{1}(k, G)$ has finite support in $G$. Given $\varepsilon>0$ choose $N$ large enough so that $\left\|f-f_{N}\right\|<\varepsilon$, and $\left|v\left(f_{N}\right)-v(f)\right|<\varepsilon$. Choose $\psi \in \Phi_{k}^{\prime}(G)$ such that $\left|\psi\left(f_{N}\right)\right|>p^{-1} v\left(f_{N}\right)-\varepsilon$. (The existence of $\psi$ follows from the first part of the argument applied to $\left\langle\operatorname{supp}\left(f_{N}\right)\right\rangle$ and 3.1.) Thus

$$
\begin{aligned}
\|f\|_{s p} & \geq|\psi(f)| \geq\left|\psi\left(f_{N}\right)\right|-\left|\psi\left(f-f_{N}\right)\right|>p^{-1} v\left(f_{N}\right)-\varepsilon-\left\|\left(f-f_{N}\right)\right\| \\
& \geq p^{-1} v(f)-p^{-1}\left|v\left(f_{N}\right)-v(f)\right|-2 \varepsilon>p^{-1} v(f)-\left(2+p^{-1}\right) \varepsilon .
\end{aligned}
$$

Since $\varepsilon$ is arbitrary, the result follows. 
The proof shows that when $\left|k^{*}\right|$ is dense in the positive real numbers, then we obtain $v(f) \leq\|f\|_{s p}$, and so in fact $v(f)=\|f\|_{s p}$.

We can now prove the following:

3.3. Theorem. Let $k$ be a complete field, $G$ an Abelian group, and $f \in$ $l_{1}(k, G)$. Then the following are equivalent:

(a) $f \in J\left(l_{1}(k, G)\right)$.

(b) $\varphi(f)=0$ for all $\varphi \in \Phi_{k}^{\prime}(G)$.

(c) $f$ is topologically nilpotent.

(d) $\varphi(f)=0$ for all $\varphi \in \Phi_{k}(G)$.

Proof.

$(a) \Rightarrow(b)$ is trivial, since $\operatorname{ker} \varphi$ is a maximal ideal of $l_{1}(k, G)$.

$(b) \Rightarrow(c)$ follows from 3.2 since (b) implies that $\|f\|_{s p}=0$.

$(c) \Rightarrow(a)$ : let $g \in l_{1}(k, G)$. Since $\left\|(g f)^{n}\right\|^{1 / n} \leq\|g\|\left\|f^{n}\right\|^{1 / n} \rightarrow 0$ we have $\left\|(g f)^{n}\right\| \rightarrow 0$, so $\sum_{n=0}^{\infty}(g f)^{n} \in l_{1}(k, G)$. In other words $1-g f$ is invertible, whence $f \in J\left(l_{1}(k, G)\right)$.

$(c) \Rightarrow(d)$ : a continuous homomorphism must map topological nilpotents to topological nilpotents, and the only topological nilpotent in a field is the zero element.

$(d) \Rightarrow(b)$ : Obvious.

The following result is useful for dealing with extensions:

3.4. Lemma. Let $G$ be Abelian, $H$ a subgroup of $G$, and let $k$ be a complete field. Assume that $H \in X_{k}$ and $G / H \in X_{E}$ for all complete extension fields $E \supseteq k$. Then $G \in X_{k}$.

Proof. Let $f=\sum_{x \in G} \alpha_{x} x \in J\left(l_{1}(k, G)\right)$. Let $\varphi \in \Phi_{k}^{\prime}(H)$, and let $\theta \in \Phi_{k}^{\prime}(G)$ be any extension of $\varphi$ (such exist by 3.1). Put $E=\theta\left(l_{1}(k, G)\right)$, a subfield of $\Omega$. Let $x \mapsto \bar{x}$ denote the natural map $G \rightarrow G / H$. If $\mu \in \Phi_{\bar{E}}(G / H)$, where $\bar{E}$ is the completion of $E$, consider the map $\psi: l_{1}(k, G) \rightarrow \Omega$ defined by $\psi(x)=\theta(x) \mu(\bar{x})$ for all $x \in G$, and extended by linearity and continuity to the whole space. Since $\psi \in \Phi_{k}(G), \psi(f)=0$ by 3.3. Put $\beta_{x}=\alpha_{x} \theta(x) \in E$, and $\gamma_{\bar{x}}=\sum_{\bar{y}=\bar{x}} \beta_{y} \in E$. In view of the definition of $\psi$ we have $0=\psi(f)=$ $\sum_{\bar{x} \in G / H} \gamma_{\bar{x}} \mu(\bar{x})=\mu\left(\sum_{\bar{x}} \gamma_{\bar{x}} \bar{x}\right)$. This is for all $\mu \in \Phi_{\bar{E}}(G / H)$, and $l_{1}(\bar{E}, G / H)$ is semisimple by assumption, so by 3.3 again, $\gamma_{\bar{x}}=0$ for all $\bar{x} \in G / H$. But $\bar{y}=\bar{x}$ is equivalent to $y \in H x$ so we have $\sum_{h \in H} \alpha_{h x} \theta(h x)=0$, where $x \in G$ is fixed. Cancelling a factor of $\theta(x)$, and remembering that $\theta$ is an extension of $\varphi$, the above becomes $\varphi\left(\sum_{h \in H} \alpha_{h x} h\right)=0$. But this is for all $\varphi \in \Phi_{k}^{\prime}(H)$, so finally every $\alpha_{h x}=0$, as required.

We need one more preliminary result.

3.5. Lemma. Let $G$ be an Abelian $p^{\prime}$-group, and let $k$ be a complete subfield of $\Omega$. Given distinct elements $x_{1}, \ldots, x_{n}$ of $G$ and elements $c_{1}, \ldots, c_{n}$ 
of $k$, there exists a continuous $k$-homomorphism $\varphi: l_{1}(k, G) \rightarrow \Omega$ such that $\left|\varphi\left(\sum_{i=1}^{n} c_{i} x_{i}\right)\right|=\left\|\sum_{i} c_{i} x_{i}\right\|$

Proof. Let $f=\sum c_{i} x_{i}$. We may assume that $\|f\|=1$. If we can find a continuous $\varphi$ such that $|\varphi(f)| \geq 1$ then the trivial fact $|\varphi(f)| \leq\|f\|$ suffices to show that $|\varphi(f)|=1=\|f\|$.

The proof of $|\varphi(f)| \geq 1$ proceeds by induction on $n$, the case $n=1$ being trivial (let $\varphi(x)=1$ for all $x \in G$ ). Assume the result is true for $n-1$ but false for $n$. Thus there exist distinct elements $x_{1}, \ldots, x_{n} \in G$, and $c_{1}, \ldots, c_{n} \in k$ with $\max _{i}\left|c_{i}\right|=1$, such that $|\varphi(f)|<1$ for all $\varphi \in \Phi_{k}(G)$ (where $f=\sum c_{i} x_{i}$ ). We may suppose $\left|c_{n}\right|=1$. It is easy to see that if $\lambda \neq 1$ is a $p^{\prime}$-power root of unity in $\Omega$ then $|1-\lambda|=1$. Since $x_{1}^{-1} x_{n} \neq 1$ we can find a $p^{\prime}$-power root of unity $\lambda \in \Omega, \lambda \neq 1$, of the same order as $x_{1}^{-1} x_{n}$. (If the order of $x_{1}^{-1} x_{n}$ is infinite let $\lambda \neq 1$ be any $p^{\prime}$-power root of unity.) Define a continuous $k$-algebra homomorphism $\varphi: l_{1}\left(k,\left\langle x_{1}^{-1} x_{n}\right\rangle\right) \rightarrow \Omega$ by $\varphi\left(x_{1}^{-1} x_{n}\right)=\lambda$. Extend this to an element (still denoted by $\varphi$ ) of $\Phi_{k}(G)$. Then

$$
\left|\varphi\left(x_{1}\right)-\varphi\left(x_{n}\right)\right|=\left|\varphi\left(x_{1}\right)\right|\left|1-\varphi\left(x_{1}^{-1} x_{n}\right)\right|=1,
$$

and so $\max _{i}\left|c_{i}\left(\varphi\left(x_{1}\right)-\varphi\left(x_{i}\right)\right)\right|=1$. By the inductive hypothesis there exists a continuous homomorphism $\mu: l_{1}(k, G) \rightarrow \Omega$ such that

$$
\left|\sum_{i=1}^{n} c_{i}\left(\varphi\left(x_{1}\right)-\varphi\left(x_{i}\right)\right) \mu\left(x_{i}\right)\right| \geq 1 \text {. }
$$

The product homomorphism $\varphi \mu$ (defined by $\left.\varphi \mu\left(\sum a_{x} x\right)=\sum a_{x} \varphi(x) \mu(x)\right)$ is also continuous, and so

$$
\left|\sum_{i=1}^{n} c_{i} \varphi\left(x_{i}\right) \mu\left(x_{i}\right)\right|<1 .
$$

Since $\left|\varphi\left(x_{1}\right)\right|=1$ we also have

$$
\left|\sum_{i=1}^{n} c_{i} \varphi\left(x_{1}\right) \mu\left(x_{i}\right)\right|=\left|\sum_{i=1}^{n} c_{i} \mu\left(x_{i}\right)\right|<1,
$$

and thus

$$
\left|\sum_{i=1}^{n} c_{i}\left(\varphi\left(x_{1}\right)-\varphi\left(x_{i}\right)\right) \mu\left(x_{i}\right)\right|<1 .
$$

This contradicts $(*)$, and proves the result.

Our main result on Abelian groups is

3.6. Theorem. Let $k$ be a complete subfield of $\Omega_{p}$, and let $G$ be an Abelian group with no $C_{p \infty}$ subgroups. Then $G \in X_{k}$.

Proof. We proceed via a series of steps.

Step 1. We may assume that $G$ is a $p$-group: If $G_{p}$ denotes the maximal $p$-subgroup of $G$ and $E$ is a complete extension field of $k$, then we claim that 
$G / G_{p} \in X_{E}$. For if $f \neq 0$ is an element of $l_{1}\left(E, G / G_{p}\right)$ then by 3.5 we can find a continuous homomorphism $\varphi \in \Phi_{E}\left(G / G_{p}\right)$ such that $|\varphi(f)|=\|f\| \neq 0$, so $J\left(l_{1}\left(E, G / G_{p}\right)\right)=\{0\}$ by 3.3. Thus if $G_{p} \in X_{k}$, then $G \in X_{k}$ by 3.4. We are now in the case where $G$ is a countable (2.2) Abelian $p$-group with no $C_{p \infty}$ subgroup.

Step 2. If $G$ has finite exponent $p^{m}$ then $G \in X_{k}$ : If $G$ has exponent $p$ then it is a countable vector space over $G F(p)$, and is therefore residually finite. Thus $G \in X_{k}$ by 2.4. In general by induction on $m$ we have $G / G^{p^{m-1}}$ and $G^{p^{m-1}} \in X_{k}$ (for all fields $k$ ), and so $G \in X_{k}$ by 3.4.

Step 3. If $G$ has no element of infinite height then $G \in X_{k}$ : The assumption is that $\bigcap_{m=1}^{\infty} G^{p^{m}}=\langle 1\rangle$, so $\left\{G^{p^{m}}: m=1,2, \ldots\right\}$ is a directed system in $G$. Each $G / G^{p^{m}} \in X_{k}$ by Step 2 , and so $G \in X_{k}$ by 2.3 .

We can now deal with countable reduced $p$-groups $G$. Consider the Ulm sequence of $G([6], \S 76)$ : put $G^{(1)}=\bigcap_{n=1}^{\infty} G^{p^{n}}$; if $\sigma$ is not a limit ordinal put $G^{\sigma+1}=\left(G^{(\sigma)}\right)^{(1)}$, and if $\lambda$ is a limit ordinal put $G^{(\lambda)}=\bigcap_{\sigma<\lambda} G^{(\sigma)}$. Since $G$ is reduced we have $G^{(\tau)}=\langle 1\rangle$ for some ordinal $\tau$. We prove, by transfinite induction on $\sigma$, that $G / G^{(\sigma)} \in X_{k}$ for all $\sigma$. For $\sigma=1$ the group $G / G^{(1)}$ has no elements of infinite height and so $G / G^{(1)} \in X_{k}$ by Step 3. If $\sigma$ is not a limit ordinal then $G^{(\sigma-1)} / G^{(\sigma)} \in X_{k}$ by Step 3, and $G / G^{(\sigma-1)} \in X_{E}$ (for all complete $E$ ) by induction, so $G / G^{(\sigma)} \in X_{k}$ by 3.4. If $\sigma$ is limit ordinal then $\left\{G^{(\rho)} / G^{(\sigma)}: \rho<\sigma\right\}$ is a directed system in $G / G^{(\sigma)}$, and since each $G / G^{(\rho)} \in X_{k}$ by induction, we have $G / G^{(\sigma)} \in X_{k}$ by 2.3. This establishes the inductive step. In particular $G=G / G^{(\tau)} \in X_{k}$, as required.

In general $l_{1}\left(k, C_{p^{\infty}}\right)$ is semisimple if and only if $k$ does not contain infinitely many $p$ th-power roots of unity ([4]; see also [1], [5]). In fact, if $k^{*}$ contains a $C_{p^{\infty}}$ subgroup then $l_{1}\left(k, C_{p^{\infty}}\right)$ even contains nonzero nilpotent elements. Thus no improvement to 3.6 without imposing additional restrictions on the field $k$ is possible and the following corollary is obvious:

3.7. Corollary. If $G$ is an Abelian group, then $l_{1}(\Omega, G)$ is semisimple if and only if $G$ does not contain a $C_{p^{\infty}}$ subgroup.

\section{SOLVABLE GROUPS}

To obtain results for solvable groups we need another extension theorem.

4.1. Lemma. Let $G$ be an Abelian $p^{\prime}$-group and suppose $\left\{x_{i}\right\}_{i=1}^{\infty} \subset G$ with $x_{i} \neq x_{j}$ for $i \neq j$. Then there exists $\varphi_{i} \in \Phi_{\Omega}(G)$ such that if $A_{n}=\left(\varphi_{i}\left(x_{j}\right)\right)_{i, j=1}^{n}$, then

$$
\left|\operatorname{det} A_{n}\right|=1 \quad \text { for all } n \text {, and }
$$

$$
\text { if } A_{n}^{-1}=\left(b_{i j}^{(n)}\right)_{i, j=1} \text {, then }\left|b_{i j}^{(n)}\right| \leq 1 \text { for } i, j=1, \ldots, n \text {. }
$$


Remark. This lemma can be compared to results on $k$-complete group algebras (c.f. [11], 4.3.3).

Proof. The proof proceeds by induction on $n$, the case $n=1$ being trivial (take $\left.\varphi_{1} \equiv 1\right)$. Assume the result holds for $n-1$, so $\varphi_{1}, \ldots, \varphi_{n-1} \in \Phi_{\Omega}(G)$ have been found satisfying properties (1) and (2). Let $\mu \in \Phi_{\Omega}(G)$ and consider the $n \times n$ matrix $M(\mu)=\left(a_{i j}\right)$ where

$$
a_{i j}= \begin{cases}\varphi_{i}\left(x_{j}\right) & \text { if } i=1, \ldots, n-1, \quad j=1, \ldots, n \\ \mu\left(x_{j}\right) & \text { if } i=n, \quad j=1, \ldots, n .\end{cases}
$$

Expanding the determinant along the $n$th row we obtain $\operatorname{det} M(\mu)=$ $\sum_{i=1}^{n} \mu\left(x_{i}\right) c_{i}$, where $\left|c_{i}\right| \leq 1$ for $i=1, \ldots, n-1$ and $c_{n}=(-1)^{n-1} \operatorname{det} A_{n-1}$. By the induction assumption $\left|c_{n}\right|=1$, thus by 3.5 there exists some $\varphi_{n} \in \Phi_{\Omega}(G)$ such that $\left|\sum_{i=1}^{n} c_{i} \varphi_{n}\left(x_{i}\right)\right|=1$. Thus (1) is satisfied. Moreover the cofactor formula for inverses makes it clear that $\left|b_{i j}^{(n)}\right| \leq 1$ for $i, j=$ $1, \ldots, n$ establishing (2).

4.2. Theorem. Let $H$ be a normal subgroup of a group $G$ and suppose $G / H$ is an Abelian $p^{\prime}$-group. If $f \in J\left(l_{1}(\Omega, G)\right)$ then $f=\sum_{i=1}^{\infty} t_{i} x_{i}$ where $t_{i} \in$ $J\left(l_{1}(\Omega, H)\right), x_{i} \in G$ and $\left\|t_{i}\right\| \rightarrow 0$.

Proof. Let $f \in J\left(l_{1}(\Omega, G)\right)$ and suppose $f=\sum_{i=1}^{\infty} f_{i}$ with $f_{i} \in l_{1}(\Omega, G)$ and supp $f_{i} \subseteq H x_{i}$ where $\left\{x_{i}\right\}_{i=1}^{\infty}$ are distinct coset representatives of $G / H$. Let $\pi: G \rightarrow G / H$ be the canonical quotient map. Apply 4.1 with the Abelian $p^{\prime}$ group being $G / H$ and $\left\{x_{i} \pi\right\}$ the distinct elements of $G / H$, to obtain $\varphi_{i} \in$ $\Phi_{\Omega}(G / H)$ with the corresponding properties (1) and (2). Extend each $\varphi_{i}$ to elements of $\Phi_{\Omega}(G)$ by defining

$$
\varphi_{i}\left[\sum_{g \in G} a_{g} g\right]=\sum_{g \in G} a_{g} \varphi_{i}(g \pi) .
$$

Define

$$
\varphi_{i}^{\#}\left[\sum a_{g} g\right]=\sum a_{g} \varphi_{i}(g) g .
$$

Then $\varphi_{i}^{*}$ is an $\Omega$-automorphism of $l_{1}(\Omega, G)$. Notice that as $\operatorname{supp} f_{j} \subset H x_{j}$

$$
\varphi_{i}^{*}(f)=\sum_{j=1}^{\infty} \varphi_{i}\left(x_{j}\right) f_{j} .
$$

Let $\varepsilon>0$ and choose $N$ such that $\left\|f_{n}\right\|<\varepsilon$ for $n>N$. Let

$$
B_{N}=\left(\varphi_{i}\left(x_{N+j}\right)\right) \quad \text { where } i=1, \ldots, N \text { and } j=1,2, \ldots \text {. }
$$

From $(*)$ we have the system of equations

$$
\left(\varphi_{i}^{\#}(f)\right)_{i=1, \ldots, N}=A_{N}\left(f_{i}\right)_{i=1, \ldots, N}+B_{N}\left(f_{i}\right)_{i=N+1, N+2, \ldots} .
$$

Hence

$$
\left(f_{i}\right)_{i=1, \ldots, N}+A_{N}^{-1} B_{N}\left(f_{i}\right)_{i=N+1, N+2, \ldots}=A_{N}^{-1}\left(\varphi_{i}^{\#}(f)\right)_{i=1, \ldots, N} .
$$


As $J\left(l_{1}(\Omega, G)\right)$ is invariant under $\Omega$-automorphisms, $\varphi_{i}^{\#}(f) \in J\left(l_{1}(\Omega, G)\right)$. Let

$$
\left(j_{i}^{(N)}\right)_{i=1, \ldots, N}=A_{N}^{-1}\left(\varphi_{i}^{\#}(f)\right)_{i=1, \ldots, N} \cdot
$$

Then $j_{i}^{(N)}$ is a linear combination of $\varphi_{1}^{\#}(f), \ldots, \varphi_{N}^{\#}(f)$ and thus belongs to the Jacobson radical for each integer $N$ and $i=1, \ldots, N$. Finally, let

$$
\left(\varepsilon_{i}^{(N)}\right)_{i=1, \ldots, N}=A_{N}^{-1} B_{N}\left(f_{j}\right)_{j=N+1, N+2, \ldots} .
$$

Then $f_{i}=j_{i}^{(N)}+\varepsilon_{i}^{(N)}$. As all the entries of $A_{N}^{-1}$ and $B_{N}$ have valuation at most one, (by the lemma) and $\left\|f_{n}\right\|<\varepsilon$ for all $n>N$, it follows that $\left\|\varepsilon_{i}^{(N)}\right\|<\varepsilon$ for all $i=1, \ldots, N$. As $\varepsilon>0$ was arbitrary we have that, for each $i$, $\lim _{n \rightarrow \infty}\left\|j_{i}^{(N)}-f_{i}\right\|=0$ and as $J\left(l_{1}(\Omega, G)\right)$ is closed $(2.1(\mathrm{iii})) f_{i} \in J\left(l_{1}(\Omega, G)\right)$. Thus

$$
f_{i} x_{i}^{-1} \in J\left(l_{1}(\Omega, G)\right) \cap l_{1}(\Omega, H) \subseteq J\left(l_{1}(\Omega, H)\right)
$$

(c.f. the proof of 2.2) and $f=\sum\left(f_{i} x_{i}^{-1}\right) x_{i}$.

The next result is obvious.

4.3. Corollary. Suppose $G_{1}$ is an Abelian $p^{\prime}$-group and $G_{2} \in X_{\Omega}$. Then $G_{1} \times$ $G_{2} \in X_{\Omega}$.

Let $S_{p^{\prime}}$ denote the class of solvable groups which have a subnormal series with Abelian $p^{\prime}$-factor groups. This class contains all solvable torsion groups which have no elements of order $p$ and all nilpotent $p^{\prime}$-groups [2].

4.4. Theorem. Suppose $H$ is a normal subgroup of $G$ with $H \in X_{\Omega}$ and $G / H \in S_{p^{\prime}}$. Then $G \in X_{\Omega}$.

Proof. Let

$$
\{1\}=K_{1} \leq K_{2} \leq \cdots \leq K_{N}=G / H
$$

be a subnormal series for $G / H$ with $K_{i+1} / K_{i}$ a $p^{\prime}$-Abelian group for each $i=1, \ldots, N$. Let $\pi^{-1}\left(K_{i}\right)=G_{i}$. We have

$$
H=G_{1} \leq G_{2} \leq \cdots \leq G_{n}=G
$$

with $G_{i+1} / G_{i} \simeq K_{i+1} / K_{i} \quad p^{\prime}$-Abelian groups, and as $H \in X_{\Omega}$ an induction argument together with Theorem 4.2 now completes the proof.

4.5. Corollary. Let $G \in S_{p^{\prime}}$. Then $G \in X_{\Omega}$.

4.6. Corollary. Let $G$ have a directed system $\left\{N_{i}: i \in I\right\}$ such that each $G / N_{i} \in S_{p^{\prime}}$. Then $G \in X_{\Omega}$.

Proof. Combine Theorem 2.3 and Corollary 4.5.

4.7. Theorem. Let $G$ have a directed system $\left\{N_{i}: i \in I\right\}$ such that each factor group $G / N_{i}$ is polycyclic. Then $G \in X_{\Omega}$.

Proof. As usual we may assume $G$ itself is polycyclic, so $G$ has a subnormal series

$$
G=G_{n} \geq G_{n-1} \geq \cdots \geq G_{0}=\{1\}
$$


with each $G_{i} / G_{i-1}$ a finitely generated Abelian group. The proof proceeds by induction on $n$. If $n=1$ the result is clear, as finitely generated Abelian groups do not contain $C_{p \infty}$. So assume $G_{i-1} \in X_{\Omega}$.

Choose a subgroup $L$ of $G$ with $G_{i} \geq L \geq G_{i-1}, G_{i} / L$ a torsion-free Abelian group and $L / G_{i-1}$ a finite Abelian group. Let $\pi: L \rightarrow L / G_{i-1}$ be the usual quotient map and assume $L / G_{i-1}=\left\{x_{i} \pi\right\}_{i=1}^{N}, x_{i} \in L$, with $x_{i} \pi$ distinct. By [11] 4.3.3 there exist homomorphisms $\varphi_{1}, \ldots, \varphi_{N} \in \Phi_{\Omega}\left(L / G_{i-1}\right)$ such that the $N \times N$ matrix $\left(\varphi_{i}\left(x_{j} \pi\right)\right)_{i, j=1}^{N}$ is nonsingular. Using this result in place of 4.1, arguments similar to those of the proof of 4.2 , (but much easier as the matrix $B_{N}$ is unnecessary) show that if $f \in J\left(l_{1}(\Omega, L)\right)$ then $f=\sum_{i=1}^{N} t_{i} x_{i}$ with $t_{i} \in J\left(l_{1}\left(\Omega, G_{i-1}\right)\right)=(0)$. Thus $L \in X_{\Omega}$. As $G_{i} / L$ is an Abelian $p^{\prime}$-group, $G_{i} \in X_{\Omega}$ (4.2). This completes the induction step and hence the proof.

\section{OPEN PROBLEMS}

1. Is $l_{1}\left(\mathbf{Q}_{p}, \oplus_{1}^{N} C_{p \infty}\right)$ semisimple? If so then it can be proved that $l_{1}\left(\mathbf{Q}_{p}, G\right)$ is semisimple for any Abelian group $G$.

2. If $G$ is a nilpotent group which does not contain a $C_{p \infty}$ subgroup, is $l_{1}(\Omega, G)$ semisimple?

3. Is the class $X_{k}$ closed under direct products or (normal) subgroups?

4. If $G \in X_{\Omega}$ and $k$ is any complete extension field of $\mathbf{Q}_{p}$ contained in $\Omega$, is $G \in X_{k}$ ?

\section{REFERENCES}

1. Y. Amice and A. Escassut, Sur la non-injectivité de la transformation de Fourier p-adique relative à $\mathbf{Z}_{p}$, C.R. Acad. Sci. Paris 278 (1974), 583-585.

2. G. Baumslag, Lecture notes on nilpotent groups, C.B.M.S. Regional Conference Series no. 2, Amer. Math. Soc., Providence, RI,1971.

3. A. Esscasut, Algebres de Banach ultramétrique et algebres de Krasner-Tate, Astérisque 10 (1973), 1-107.

4. J. Fresnel and B. Mathan, Algebres $L^{1} p$-adiques, Bull. Soc. Math. France 106 (1978), 225-260.

5. __ Sur la transformation de Fourier p-adique, C.R. Acad. Sci. Paris 277 (1973), 711-714.

6. L. Fuchs, Infinite abelian groups, Academic Press, New York, 1973.

7. P. Kropholler, P. Linnell, and J. Moody, Applications of a new K-theoretic theorem to solvable group rings, Proc. Amer. Math. Soc. 104 (1988), 675-684.

8. R. Larsen, Banach algebras, Marcel Dekker Inc., New York, 1973.

9. J. Lawrence, The Jacobson radical of tensor products, 1989 (preprint).

10. M. Parmenter and S. Sehgal, Non-archimedean group algebras, J. Number Theory, 7 (1975), 376-384.

11. D. Passman, The algebraic structure of group rings, John Wiley and Sons, New York, 1977.

12. A. C. M. van Rooij, Non-archimedean functional analysis, Marcel Dekker Inc., New York, 1978.

13. I. E. Segal, The group algebra of a locally compact group, Trans. Amer. Math. Soc. 61 (1947), 69-105. 
14. A. Strojnowski, A note on u.p. groups, Comm. Algebra 8 (1980), 231-234.

15. J. Tate, Rigid analytic spaces, Invent. Math. 12 (1971), 257-289.

Department of Pure Mathematics, University of Waterloo, Waterloo, Ontario N2L 3G1 CANADA

Department of Mathematics, University of Alberta, Edmonton, Alberta T6G 2 G 1 CANADA 